\title{
Determining the Weights of the Value Factors of Used Cars and the Example of Evaluating Panxi Used Cars
}

\author{
Jun $\mathrm{Li}^{1,}$, JianChun Gong ${ }^{2, b}$ and Lin Peng ${ }^{3, \mathrm{c}}$
}

${ }^{1}$ Sichuan Electromechanical Institute of Vocation and Technology Sichuan, 617000, P.R. China

2 Panzhihua University, Sichuan, 617000, P.R. China ull address of second author, including country

${ }^{3}$ Panzhihua Technician College, Sichuan, 617000, P.R. China

a419755313@qq.com, bjcgong01@163.com,, c1016436691@qq.com

Keywords: Into the new rate, replacement costs, depreciation of the law, Panxi area, Used Cars Abstract. In the used car assessment process affect the value of used car The determination of the weight of each factor is very important, the weight used to determine the actual rate of the used car was evaluated into the actual rate into the new cost is the evaluation value, a direct impact on secondThe interests of both sides, affecting the healthy development of used car industry. China's used car market mostly use the oldest method of depreciation method, and then according to the car and the appearance of used car to determine the transaction price, so the evaluation of used car prices are very rough. This paper determines the weight of each influencing factor, and then calculates the actual rate to determine the price. Finally, the use of Panxi area used car example illustrates the feasibility of its method.

In the used car in the process of evaluation weights of each factor to determine the value of used car is very important in determining the weight of assessment of the actual used car into a new rate, the actual rate of replacement cost is a new evaluation value, directly affect the used car trading interests of both sides, affecting the healthy development of the used car industry. The used car market in China mostly adopts the oldest method, the depreciation method, and then determines the transaction price of used cars according to their condition and appearance, so that the price of used cars is very rough. This section defines the weights of each factor in the previous chapter, and then calculates the actual rate of innovation to determine the evaluation price. Finally, an example is given to illustrate the feasibility of the method.

\section{determination of replacement cost}

Determine the replacement cost is used car evaluation institutions do, must be scientific, reasonable, fair and impartial, without considering the evaluation of any information vehicles, there is a unified method of determination [7]. At present, there are two ways to determine the replacement cost, one is property right change, and another is ownership transfer [1]. The two way is slightly different, the former one should consider various kinds of taxes, the latter is the price on the existing market. As a result of the general situation, the used car transaction is the property right change, this article studies is the property right change form. Formula for replacement cost:

$$
Q=A+\frac{A}{1+17 \%} \times 10 \%
$$

Type: Q - replacement cost;

A - New car prices.

\section{Brief introduction of analytic hierarchy process}

Analytic hierarchy process (abbreviated as AHP) was formally proposed by the United States in 1970s by Professor T.L.Saaty, an American operational research expert. It combines quantitative research with qualitative research, and divides the problems into hierarchical [10]. Its characteristic is to make complex problems orderly and organized. AHP can determine the impact factors, stratify the 
impact factors, and then rank the importance of each layer to determine the degree of importance of each factor [11]. The analytic hierarchy process (AHP) can be divided into three levels: target layer, criterion layer and scheme layer. Because people are limited to data, they can not be compared beyond a certain limit. Based on the limited ability of human beings, Professor T.L.Saaty, an American research expert, proposes the 1 - to 9 - scale [12], which is shown in table 1 below.

Table1 scale nine and Its Implications

\begin{tabular}{|c|c|}
\hline Scaling & Definition \\
\hline 1 & Compared to A, I is as important as J \\
\hline 3 & Compared to A, I is more important than $\mathrm{j}$ \\
\hline 5 & Compared to A, I is more important than $\mathrm{j}$ \\
\hline 7 & Compared to A, I is much more important than $\mathrm{j}$ \\
\hline 9 & Compared with A, I is more important than $\mathrm{j}$ \\
\hline $2,4,6,8$ & Relative to A, the extent of I over J is between the above two \\
\hline $\begin{array}{c}\text { The reciprocal of } 1- \\
9\end{array}$ & $\begin{array}{c}\text { In contrast to A, the scaling factor representing the factor I versus factor } \mathrm{j} \text { is equal to the } \\
\text { reciprocal of the scale value of the factor J and factor I }\end{array}$ \\
\hline
\end{tabular}

\section{Into a new rate of research}

\section{basic innovation rate}

According to service life was determined based on a new method to calculate the rate, the new rate is very rough, without considering any link in the process of using, such as operating environment, use intensity, nature of work. So we must revise the basic rate and determine its adjustment factors.

\section{adjustment factor}

Based on the analysis of the fourth section, the adjustment factors for the basic rate of innovation are divided into five categories: technical performance, technical status, hedge ratio, and other adjustment factors. In these four categories, there are many factors in each category. If the general method is not able to distinguish who is important and who is not important, this paper uses the analytic hierarchy process to determine the importance.

Determine and calculate the weight of each factor adjustment factor for the performance of Technology.

Hierarchical structure diagram of technical performance adjustment factors 1 .

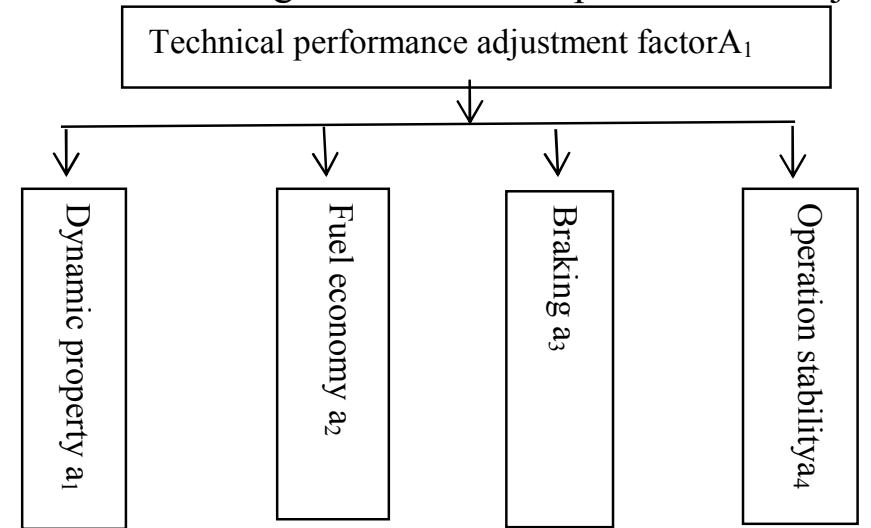

Figure 1 hierarchical structure of technical performance adjustment factors

1) Technical performance ${ }^{A_{1}}$ _a Judgment matrix

Technical performance is very important for a car, to a certain extent, determines the quality of the car. According to the Panzhihua terrain features, consumers of power requirements are higher, and consumers of used car maintenance costs and vehicle safety very seriously, and on the fuel economy of automobile braking and higher requirements. In conjunction with the Panzhihua used car ppraiser's recommendations, determine technical performance ${ }^{A_{1}}$ - a the judgment matrix is shown in table 2 below. 
Table $2 A_{1}$ - a judgment matrix

\begin{tabular}{|c|c|c|c|c|c|}
\hline A1 & $\mathrm{a} 1$ & $\mathrm{a} 2$ & $\mathrm{a} 3$ & 3 & \\
\hline $\mathrm{a} 1$ & 1 & 3 & & 2 & 1 \\
\hline $\mathrm{a} 2$ & $1 / 3$ & 1 & & 1 & 3 \\
\hline $\mathrm{a} 3$ & $1 / 3$ & $1 / 2$ & & $1 / 3$ & 3 \\
\hline $\mathrm{a} 4$ & 1 & $1 / 3$ & & 1 \\
\hline
\end{tabular}

Calculated by Matlab:

In the result of calculation, the diagonal of the matrix $\mathrm{D}$ is the eigenvalue, and the column vector of the matrix $\mathrm{X}$ pair is the characteristic vector, as shown in figure 3.2. The rest of the process is as follows, the legend is skipped.

Maximum eigenvalue $\lambda_{\max }=4.7172$

Feature vector $\mathrm{W}=(0.7510,0.4823,0.3558,0.2771)^{\mathrm{T}}$

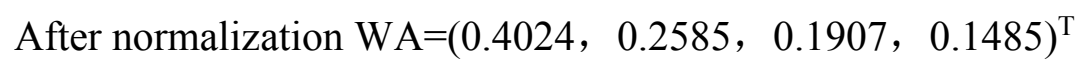

The weight of the A to the A1 is calculated as follows

$a_{1}$ - Power adjustment factors accounted for the weight of technical performance 0.4024

$a_{2}$ _the fuel economy adjustment factor accounts for 0.2585 of the technical performance

$a_{3}$ _the braking adjustment factor accounts for 0.1907 of the technical performance

$a_{4}$ _the operating stability adjustment factor accounts for 0.1485 of the technical performance

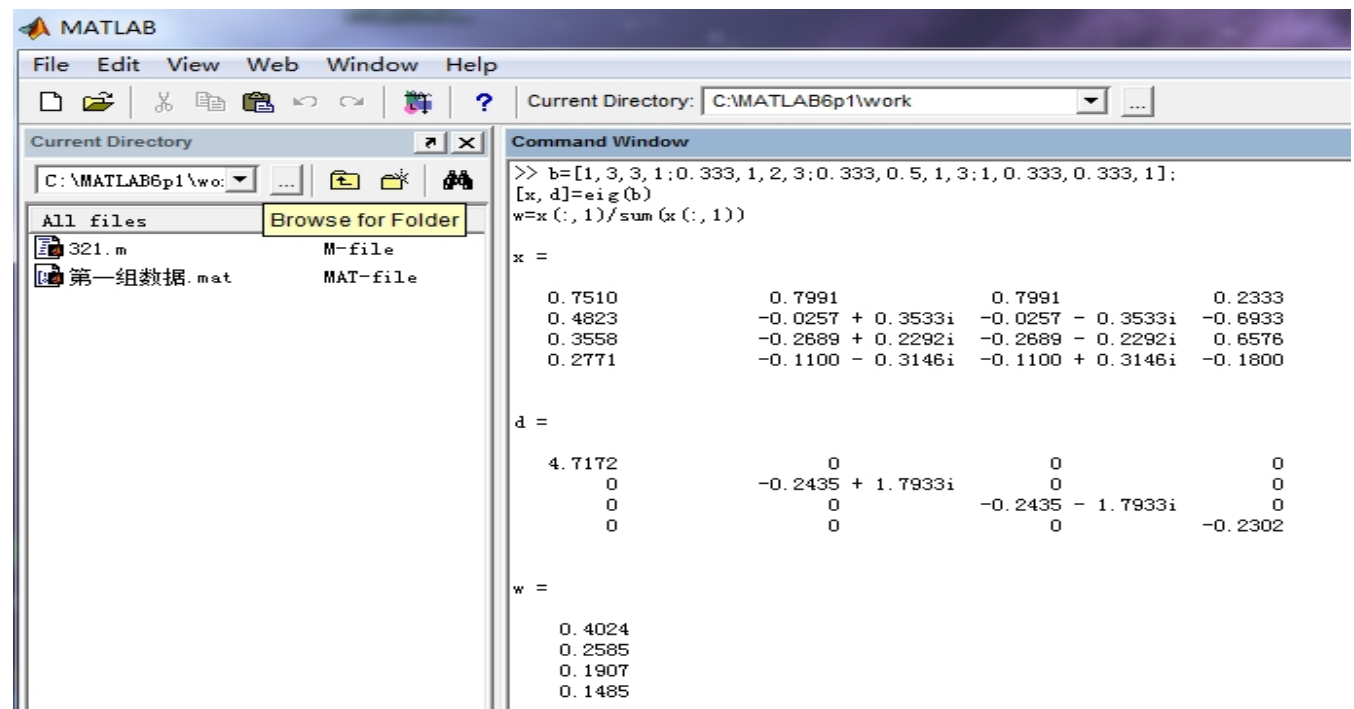

Figure 2 judgment matrix Matlab calculation results

2) A formula for calculating the technical performance adjustment factor A1

$$
A_{1}=J_{1} \times a_{1}+J_{2} \times a_{2}+J_{3} \times a_{3}+J \times a_{4}
$$

Type: $A_{1}$ - technical performance adjustment factor

$J_{1}$ _Used vehicle dynamic quantitative index

$J_{2}$ _Used car fuel economy quantitative index

$J_{3}$ _ Used vehicle brake quantitative index

$J_{4}$ _Used vehicle operation stability quantitative index

$a_{1}$ _ power adjustment factor, which is the weight of technical performance

$a_{2}$ _Fuel economy adjustment factor, the weight of technical performance 
$a_{3}$ _the braking adjustment factor accounts for the weight of the technical performance

$a_{4}$ _operating stability adjustment factors, accounting for technical performance weights

The adjustment of technical status and the determination and calculation of the weight of each factor.The most important factor in the adjustment of the technical condition is the vehicle heart engine, which provides the power for the whole vehicle. Second, consumers are very concerned about the main passive security, the most active safety considerations is the braking performance. Body and interior affect beauty, this is also a consumer concern. The running system and transmission system have a great influence on the operation stability of the vehicle. According to the appraiser's suggestion and the relevant data, the judgment matrix is shown in table.

Calculated by Matlab: As described above, omitted.

\section{Summary}

China's used car industry is in the initial stage compared with developed countries, there are a series of problems, such as the system is not perfect and so many problems that hinder the healthy development of used car industry, but hinder the development of used car industry should be the most important evaluation system is not perfect, not reasonable, are more subjective, so down the same a used car appraiser of different evaluation results may be different, and a larger gap. This article aims at these problems and establishes an objective quantitative evaluation system.

1) first introduced the domestic and foreign second-hand vehicle profession development present situation, and analyzed our country and the overseas difference.

2) analyze the advantages and disadvantages of the replacement cost method, the current market price method, the liquidation price method, and the present value method of income. Finally, it is determined that the replacement cost method is suitable for the current domestic situation.

3) now many assessment institutions adopt the fixed number of year depreciation method, and the same result is obtained for different conditions. This is certainly unreasonable and unscientific. This is based on the depreciation method, which is adjusted by a series of adjustment factors. The weights of each adjustment factor are determined by analytic hierarchy process (AHP), and the adjustment coefficients are finally determined.

4) finally, the rationality of the evaluation system is verified by a case. There is little difference in vehicle pricing between the vehicle and the current market through the system.

\section{References}

[1] Huang Qiuping. Selection and comparison of appraisal methods for used cars. Traffic world, 2012.

[2] Wang Qin, Wu Kelin, Du Shidong, Liang Hongming. Replacement cost method for value assessment of used cars. Automotive and accessories, 2010

[3] Pang, Changle. Practice and evaluation of used car. First edition. Beijing Institute of Technology press, 2007

[4] Yu Yu. Automotive theory. Fifth edition. China Machine Press,.2015

[5] Wang Zhihong, Liu Chengwu. Automotive inspection and maintenance. First edition. People Communications Press, 2013

[6] Yang Yuanlei. Research on the evaluation method of old motor vehicle based on the replacement cost method [D]. Chang'an University, 2012

[7] Travel. Study on value evaluation method of used car based on replacement cost method [D]. Liaoning University of Technology, 2014.

[8] Guo Zhenjiang. Research on the establishment and system of evaluation methods for old motor vehicles. Master's degree thesis of Chang'an University, 2011

[9], Gospdinov, Nikolay, Kan, Raymond, Robitti, Cesare. Ch-squared, tests, for, evaluation, and, comparison, of, asset, pricing, models. Journal, of, Econometrics. 
[10], Guo Jinyu, Zhang Zhongbin, Sun Qingyun. Research and application of analytic hierarchy process. Chinese Journal of safety science, 2008

[11], Miroslaw, kwiesielewicz, Ewa, Van, Uden. Inconsistent, and, contradictory, judgments, in, pairwise, comparison, method, in, the, AHP. Computers \&amp; amp: Operations, Research.

[12], Luo Zhengqing, Yang Shanlin. Comparison of several scales in analytic hierarchy process. Systems engineering theory and practice, 2006 